\title{
Correction to: Long-Term Recovery of Microbial Communities in the Boreal Bryosphere Following Fire Disturbance
}

\author{
Nick A. Cutler ${ }^{1,2}$ - María Arróniz-Crespo ${ }^{3}$ - Lorna E. Street ${ }^{4} \cdot$ David L. Jones $^{3} \cdot$ Dominique L. Chaput $^{5}$. \\ Thomas H. DeLuca ${ }^{6}$
}

Published online: 8 July 2019

(C) Springer Science+Business Media, LLC, part of Springer Nature 2019

\section{Correction to: Microbial Ecology (2017) 73:75-90} https://doi.org/10.1007/s00248-016-0832-7

The original version of this article contained an error in the Molecular Analysis subsection of the Methods. In this subsection, the sequence for the primer CYA781R was specified incorrectly. The text should read: "To check the analysis, a second pyrosequencing assay was performed using a different moss shoot sample and a primer set targeted at Cyanobacteria, i.e., CYA359F (5'-GGGGAATYTTCCGCAATGGG-3') and 781R (5'-GACTACWGGGGTATCTAATCCCWTT-3')." The correct primer set was used during the analysis, so our findings are unaffected. The authors apologize for this error.

The online version of the original article can be found at https://oi.org/ 10.1007/s00248-016-0832-7

Nick A. Cutler

nick.cutler@ncl.ac.uk

1 Scott Polar Research Institute, Lensfield Road, Cambridge CB2 1EP, UK

2 School of Geography, Politics and Sociology, Newcastle University, Newcastle NE1 7RU, UK

3 School of Environment, Natural Resources \& Geography, Bangor University, Bangor, Gwynedd LL57 2UW, UK

4 Terrestrial Environmental Sciences, Heriot-Watt University, Edinburgh EH14 4AS, UK

5 Department of Mineral Sciences, Smithsonian Institution, National Museum of Natural History, 10th \& Constitution NW, Washington, DC 20560-119, USA

6 School of Environment and Forest Science, University of Washington, Seattle, WA 98195, USA 